EESTI NSV TEADUSTE AKADEEMIA TOIMETISED, 27. KOIDË

FOOSIKA * MATEMAATIKA. 1978, NR. 4

ИЗВЕСТИЯ АКАДЕМИИ НАУК ЭСТОНСКОП ССР. ТОМ 27 ФИЗИКА * МАТЕМАТИКА, 1978, № 4

\title{
НАУЧНО-ТЕХНИЧЕСКАЯ ПРОГРАММА АКАДЕМИИ НАУК ЭССР ПО ВЫЧИСЛИТЕЛЬНОЙ ТЕХНИКЕ
}

\author{
(Представлена Н. Алумяэ)
}

\section{1. Предпосылки Программы}

В начале и середине 60-х годов - в пернод универсальных ЭВМ второго поколения - начали развиваться специализированные системы программирования, ориентированные на конкретные группы потребителей и предназначенные для решения определенных, четко детерминированных классов задач.

В эту новую область математического моделирования и системного программирования активно включился Институт кибернетики Академии наук ЭССР. Объектами его исследования стали программные методы управления различными металлорежущими станками с цифровым программным управлением (ЦПУ). В результате этого были разработаны и успешно внедрены на крупных заводах страны система САП-2 [ ${ }^{1,2}$ ] для управления фрезерными, а спустя три года - система АПРОКС для управления газорезательными станками с ЦПУ $\left[{ }^{3}\right]$. Последняя система используется в судостроительной промышленности и сейчас. Помимо этого, все бо́льшую актуальность стали приобретать проблемы автоматизации экономических расчетов, где также требовались аналогичный подход и легко осваиваемый язык программирования как инструмент для потребителя.

Исследования в этом направлении увенчались созданием анкетной системы обработки экономических данных, работающей на быстром трансляторе ВЭЛГОЛ, которая получила широкое распространение в нашей стране $\left[{ }^{4}\right]$.

Bce эти системы снабжены мнемоническими языками высокого уровня, ориентированными на потребителя без программистского образования, но являющегося специалистом в области, для которой предназначена данная система $\left[{ }^{5}\right]$. Благодаря возможности описывать в текстах программ свои задачи на т. н. проблемном уровне, не вдаваясь в сложный механизм программирования вычислительных процедур, определения структур данных, распределения запоминающих устройств и т. д., указанные системы быстро завоевали популярность среди потребителей и нашли у них дальнейшее развитие $\left[{ }^{6,7}\right]$.

Накопленный опыт позволил создать в первой половине 70 -х годов в Институте кибернетики интегрированную систему программирования (ИСП) также с проблемной ориентацией. В основу этой работы был 
положен принцип, заключающийся в том, что комплексные инженернотехнические задачи вначале подвергаются декомпозиции на отдельные подзадачи, которые затем решаются группами различных специалистов (часто различных специальностей и не только на одном уровне), а окончательное решение получается в результате согласования промежуточных результатов, дополнительного обмена информацией и данными, изменения отдельных локальных целей. Естественно, необходнмо гарантировать сходимость этого процесса, чтобы получить желаемое решение комплексной задачи. Поэтому ИСП состоит из открытого множества спещиализнрованных подсистем, совместная работа которых обеспечивается системным процессором автоматически. Кроме того, ИСП снабжена средствами саморазрешения, т. е. она является метасистемой, с помощью которой определяются и генерируются новые специализированные подсистемы или (и) части, входящие в ее состав $\left[{ }^{8-13}\right]$.

Bce указанные системы работают на универсальных ЭВМ и предназначены для решения задач, не требующих прямого он-лайн управления вычислениями в реальном масштабе времени, т. е. для решения, например, задач подготовки производства, инженерного и экономического планирования, машинного проектирования и т. д.

При переходе к задачам прямого управления надо иметь в виду, что построенне системы управления (СУ) сложными (быстро меняющимися, большой размерности) техническими объектами порождает комплекс проблем, соразмерный по сложности с управляемым объектом. СУ должна быть неотъемлемой частью объекта, работать в темпе его функционирования, быть гибкой и надежной. Такими свойствами могут обладать правнльно сконструнрованные цифровые системы обработки информацин, связанные с объектом с помощью нзмерительных и управляемых каналов. Специализация СУ на уровне технических средств и программного обеспечения требует, в свою очередь, детального исследования класса объектов и наличия универсальных средств проектирования этих систем.

Весьма характерными объектами управления являются технологические процессы непрерывного или циклического типа, которые в течение ряда лет питали исследования по методам управления в разных научных центрах Эстонской ССР.

Уровень развития вычислительной техники оказал существенное влияние на характер этих работ. В 60-е годы орнентация на универсальные ЭВМ вынуждала ограничиваться объектами с медленно изменяющимися процессами, хотя, с другой стороны, позволяла концентрировать внимание на сложных моделях $\left[{ }^{14},{ }^{15}\right]$. В этот период для решения задач моделирования и оптимизацин отдельных процессов металлургии и химической технологии использовались результаты работ Института кибернетики по математической статистике и вычислительной математике. Была разработана и внедрена на многих химических заводах централизованная система ФОРСАЛ для управления процессом производства формальдегида. На универсальной $Э В \mathrm{~B}$ вычислялись режимы сравнительно несложных и медленно меняющихся процессов $[16]$.

В конце 60-х годов в результате обобщения решаемых проблем начали развиваться работы по теорни управления стохастическими объектами. В качестве моделей рассматривались управляемые случайные процессы. Разрабатывались методы дуального управления дискретными и непрерывными случайными процессами, методы планирования экспериментов, методы стохастического математического программирования и математического программирования при неопределенности [ $\left.{ }^{16-22}\right]$. 
Однако и здесь ограниченные возможности вычислительной техники, а именно отсутствие он-лайн связи с объектом, тормозили практическую реализацию этих методов в СУ.

Предпосылки для построения СУ реального времени появились в начале 70-х годов с распространением управляющих ЭВМ третьего поколения. Открылась техническая возможность построения он-лайн систем сбора информации и управления для сложных объектов, хотя необходимость подчинения моделей и алгоритмов управления скромным вычислительным возможностям этих ЭВМ осталась. На первый план встал учет сложных взаимосвязей управляемых объектов. Были развиты работы по цифровому многосвязному регулированию линейных систем, имитационному моделированию и управлению объектов с сетевой структурой $\left[{ }^{23,24}\right]$. Проблемы моделирования сложных объектов исследовались в Институте кибернетики, Таллинском политехническом институте и Электротехническом НИИ (ныне Научно-исследовательский и проектно-технологический институт систем планирования и управления в электропромышленности Таллинского электротехнического завода им. М. И. Калинина) [ $\left.{ }^{25}\right]$. Получили развитие работы по программному обеспечению управляющих ЭВМ как операционными системами, так и пакетами прикладных программ $\left[{ }^{26,27}\right]$.

K созданию специализированных ЭВМ приступили в Электротехническом НИИ. Здесь были разработаны и изготовлены специализированная ЭВМ СТЭМ и ее программное обеспечение для расчета режимов резания и норм времени для металлорежущих станков $\left[{ }^{28}\right]$. На ней решаются задачи оптимизации величин скорости резания и стойкости инструмента при большом числе нелинейных и дискретных ограничений. Целевая функция при оптимизации - минимизация расходов при обработке. К системе было предъявлено также требование - работать непосредственно в технологических бюро заводов без какой-либо дополнительной аппаратуры для подготовки исходных данных.

В результате была создана серия малых ЭВМ, специализация которых заключалась в следующем. Во-первых, наличие весьма большого для того времени постоянного запоминающего устройства емкостью 32000 слов для хранения программ. Во-вторых, реализация специализированного входного языка для ввода в машину оперативных данных и его программно-аппаратного интерпретатора. В третьих, применение специальных команд, особенно команды поиска данных из таблиц.

В результате такой специализации эффективность ЭВМ по сравнению с универсальными ЭВМ того времени резко возросла. При скорости процессора примерно в 3 раза ниже задачи решались приблизительно в 20 раз быстрее и в 6 раз дешевле, чем на универсальной $\ni B M$.

Опыт, накопленный при использовании этих машин на восьми машиностроительных заводах страны, убедительно показал высокую эффективность проблемно-ориентированных систем в области технологии машиностроения. $\mathrm{K}$ сожалению, широкое производство этих машин не было организовано, хотя спрос на них весьма высок и сегодня. Поэтому вопрос их производства (разумеется, на современном уровне аппаратуры) предусмотрен рамками Программы.

Параллельно с перечисленными работами велись исследования по модульному программированию. В результате были созданы система модульного программирования (СМП) и пакет решения инженерных задач (ПРИЗ). В этих системах была впервые применена современная технология программирования, аналогичная т. н. НІРО. Согласно этой технологии, разработку программ начннают с описания и документи- 
рования функций и структуры данных сверху вниз. В результате этого уже на первых этапах разработки программ получают документы, являющиеся по существу сборочным чертежом программы и содержащие предварительные описания всех ее компонентов. На основе такой документации программа любой сложности разрабатывается аналогично инженерному проекту. Основным достоинством такого метода программирования является получение качественных и хорошо отлаживаемых программ за такие короткие сроки, которые недостижимы никакими другими способами.

\section{2. Программа по вычислительной технике}

В 1975 г. в Институте кибернетики пришли к выводу, что необходимо объединить математиков, программистов и специалистов по аппаратуре вычислительной техники с тем, чтобы более укрупненными силами и по единой программе планировать дальнейшие исследования и практические работы. Это решение соответствовало общему направлению развития вычислительной науки, вычислительной техники и их использованию во всессюзном и международном масштабах. Предложение института о составлении республиканской Программы по исследованию, разработке и использованию малых ЭВМ с проблемной ориентацией было обсуждено и одобрено как на бюро Отделения физико-математических и технических наук, так и в Президиуме АН ЭССР.

Институт кибернетики располагал относительно сильными кадрами по программированию, однако его материально-техническая база средств вычислительной техники, а также работы по электронике были менее развиты. Поэтому создание соответствуюшего конструкторского бюро стало первоочередной задачей. Один из секторов института был реорганизован в сектор инструментального математического обеспечения, создан сектор систем управления. Ведущие специалисты Таллина, работавшие до этого малочисленными группами в различных организациях, были привлечены к работам по новой Программе. Установились координированные связи на новом и конкретном уровне с СКБ АН ЭССР, с Таллинским политехническим институтом, с рядом организаций других братских республик и, разумеется, с головной организацией по разработке малых ЭВМ - Институтом электронных управляющих машин, Таллинское отделение которого также работает при Институте кибернетики. В настоящее время Программа охватывает двести сотрудников, среди них - три доктора и около тринадцати кандидатов наук.

При определении целей Программы была поставлена задача - одновременно получить научные, технические и практические результаты, т. е. создать комплексную программу.

Н аучны е цели Программы - дальнейшее развитие основ эффективного проектирования специализированных вычислительных устройств и систем реального времени, а также соответствующего математического обеспечения. Для достижения этих целей предусматриваются следующие научно-исследовательские работы:

- развитие теории проблемно-ориентированных вычислительных структур и децентрализованных систем на основе наиболее перспективной элементной базы - микропроцессоров;

- развитие теории алгоритмического описания, моделирования и анализа систем реального времени; разработка соответствующих методик для широкого класса СУ;

- разработка средств и методики контроля и диагностики ненсправно- 
стей вычислительных систем, а также способов обеспечения их надежности и живучести в работе;

- развитие систем машинного проектирования.

Т ехнически ц цели Прогр ам мы - разработка и конструирование в рамках Программы малых ЭВМ проблемно-ориентированных систем реального времени, а также создание средств и технологии изготовления вычислительной аппаратуры. В частности, предусматриваются:

- разработка функциональных модулей систем реального времени;

- создание комплекса средств инструментального программирования для разработки на больших универсальных ЭВМ математического обеспечения малых вычислительных систем;

- разработка и составление технологических процессов пронзводства вычислительной аппаратуры.

Пр актически ецели Программы:

- создание научно-технических предпосылок для организации в XI пятилетке в Эстонии промышленного производства вычислительной аппаратуры и математического обеспечения;

- создание в Институте кибернетики экспериментального производства для изготовления опытных партий аппаратуры и СУ, в том числе средств для автоматизации научного эксперимента;

- проектирование и изготовление конкретных СУ для народного хозяйства республики.

\section{3. Основные направления работ по Программе}

Реализация Программы идет по пути разработок конкретных систем и устройств. Одним из важнейших направлений является а в т ом а т и за ция проектирования математического обеспечения АСУТП. Имеющийся опыт реализации промышленных АСУТП подсказывает следующие подходы к решению этой проблемы:

- исследование общих свойств классов сбъектов управления;

- создание оптимальных алгоритмов управления;

- создание пакетов прикладных программ для их реализации;

- создание специализированных операционных систем реального времени для универсальных управляющих ЭВМ или ЭВМ, специализированных для объектов определенного класса;

- создание инструментальных систем программирования для управляющих ЭВМ;

- создание специализированных периферийных устройств, в частности диалоговых средств для аппаратов СУ.

Примером реализации такого комплексного подхода является экспериментальная СУ непрерывным химическим процессом на базе универсальной управляющей малой ЭВМ ЕС-1010, уже внедренная в промышленную эксплуатацию.

В системе реализованы разработанные в Институте кибернетики методы имитационного моделирования химико-технологических систем, многосвязного цифрового регулирования, генерирования программ сбора и первичной обработки информации, специализированная для данного класса задач операционная система, программы диалога с оператором. Разработан пульт оператора АСУТП и устройства сопряжения с объектом.

Одномашинная многофункциональная АСУТП имеет ряд недостатков, в первую очередь - сложность программного обеспечения, что приводит к трудностям отладки системы, высокой цене, малой надежности и длительным срокам проектирования. 
Рассредоточение системы путем использования взаимосвязанных микро-ЭВМ допускает узкую специализацию любой из них. Реализация же каждой возможности требует комплексного решения ряда проблем по численным методам, программному обеспечению и техническим средствам.

Применение микро-ЭВМ означает дальнейшее уменьшение вычислительных возможностей отдельной ЭВМ и, как следствие, предельное упрощение алгоритмов. Алгоритмы управления для крупных объектов могут быть реализованы путем параллельного решения совокупности малых задач, таких, как сбор данных, идентификация и имитация объекта, оптимизация и многосвязное цифровое регулирование.

В целях реализации идей децентрализации разрабатываются м а лые системы для управления отдельными агрега тами электростанций на базе микро-ЭВМ. Они, при условиях легкой взаимозаменяемости и соединения в общую иерархию управления, позволяют создавать высоконадежные живучие системы при умеренных требованиях к надежности отдельных ЭВМ.

Практически это ведет к замене традиционной автоматики малыми ЭВМ, а это позволяет:

- значительно экономить рабочие площади электростанций, ибо средства традиционной автоматики занимают залы площадью в несколько сот квадратных метров;

- облегчать процесс проектирования и производства средств автоматики, ибо переход от агрегата к агрегату состоит в замене только части программного обеспечения.

Первая такая система разрабатывается для управления газотурбинной установкой мощностью $20 \mathrm{MBT}$, применяемой на пиковых электростанциях. Она позволит получить практический опыт и проверить теоретические концепции.

Замена традиционных средств автоматики сетью микро-ЭВМ порождает, однако, свои проблемы. Основными из них являются распределение обязанностей между отдельными ЭВМ и обучение проектировщиковавтоматчиков новым средствам.

Распределение функций между отдельными ЭВМ сети - задача компромиссная. Здесь надо учитывать следующие параметры и характеристики отдельных процессов:

- количество входных и выходных снгналов;

- наличие и количество сигналов, единых для нескольких процессов;

- количество общих данных, относящихся как к процессам, так и к организационным программам;

- функции операционных систем отдельных микро-ЭВМ;

- способы коммуникации между отдельными микро-ЭВМ;

- функциональная целостность и возможность автономного тестирования и ввода в действие отдельных ЭВМ.

Проблема переквалификации проектировщиков-автоматчиков, использующих средства вычислительной техники, является более серьезной, так как касается широкого круга специалистов и требует поэтому больших затрат времени. Обучение всех проектировщиков программированию трудная и, видимо, нереальная задача; более перспективным для решения этой проблемы представляется путь создания организационных и технических средств, облегчающих постановку и программирование задач. Сюда относятся: методики строгого описания задач в инженерных терминах, проблемно-ориентированные языки программирования, системы машинного проектирования и библиотеки типовых программных модулей. 
Опыт, полученный при решении этих задач, станет особенно ценным в ближайшие годы, когда системы на базе микропроцессоров найдут массовое применение во многих устройствах автоматики.

Производство программного обеспечения малых ЭВМ является существенной частью Программы. Изменение функций малых ЭВМ, в частности переход к использованию микропроцессоров и малых ЭВМ, потребовало перестройки всего производства программ. Основным методом стало их машинное проектирование, выполняемое на достаточно мощных инструментальных вычислительных машинах серии ACBT и EC. Обычно программа как компонент вычислительной системы создается одновременно с разработкой аппаратуры, поэтому распространенным подходом стало изготовление и полная отладка программ на базовой машине. Применяется техника кросс-компиляции, когда компилятор для объектной малой ЭВМ работает на мощной инструментальной ЭВМ и выдает программу, готовую к пуску на объектной $Э \mathrm{BM}$.

Преимущества такого подхода:

- возможность параллельной разработки программ и аппаратуры;

- возможность построения качественной инструментальной системы программирования на мощной ЭВМ;

- универсальность инструментальной системы программирования, позволяющая быстро переходить к разработке программ для новых ЭВM;

- низкие эксплуатационные расходы инструментальной системы на универсальных серийных ЭВМ.

Традиционные методы автоматизации программирования основаны на переводе программы с одного языка на другой, что позволяет человеку пользоваться алгоритмическими языками высокого уровня [ $\left.{ }^{29}\right]$. Новым направлением в машинном проектировании стало конструирование программ по содержательному описанию задач. В этом направлении достигнуты уже практические результаты. Автоматический синтез частей программ осуществлен в системе ПРИЗ. Развитие работ этого направления должно уже в ближайшие годы обеспечить высокий уровень автоматизации программирования систем малых ЭВМ. Практическая реализация систем программирования для них требует решения ряда дополнительных проблем, в частности необходима более точная привязка программ к аппаратуре, а также, учитывая ограниченные возможности аппаратуры, более полная оптимизация программ по быстродействию и использованию памяти.

Большая гибкость современных вычислительных систем, содержащих микропроцессоры, открывает совершенно новые перспективы перед разработчиками. Появляется возможность аппаратной реализации модулей. Это, в свою очередь, позволит распараллелить вычисления по отдельным модулям. Встает практическая задача управления параллельными вычислениями, которую до сих пор нельзя считать решенной, несмотря на значительное число работ в этом направлении.

Аппаратная реализация функциональных модулей приближает проектирование программ к проектированию аппаратуры. Возникает потребность в создании ИСП вычислительных систем. Общая методика проектирования в таких системах может базироваться на нисходящем модульном проектировании, как в методике НIPO.

На верхних функциональных уровнях проектирование программ и проектирование аппаратуры не различаются. Разделение начинается только с определенного уровня детализации и идет в нескольких направления $\mathrm{x}$ : 
- программирование на алгоритмических языках,

- программирование микропроцессоров,

- микропрограммирование,

- проектирование аппаратуры.

Благодаря появлению интегральных схем и автоматизации их проектирования разработка цифровых схем и узлов ӘВМ все более упрощается, в то время как проверка правильности работы и диагностика неисправностей, напротив, во многих случаях усложняются из-за отсутствия доступа к отдельным элементам, находящимся внутри интегральной схемы. Поэтому вопросы контроля и ди агностики неи с правностей ци фровых схем останутся актуальными, очевидно, еще длительное время, их решение является также одной из целей Программы.

На базе новых технических средств (малая ЭВМ, устройства связи и т. д.) и проблемно-ориентированного математического обеспечения создается система комплексной проверки и диагностики как нескомпонованных печатных плат, так и цифровых схем. Проверяемыми цифровыми схемами являются модули малых ЭВМ - устройства арифметики, устройства управления, карты интерфейса и т. д.

В области проверки и диагностики имеется ряд нерешенных теоретических проблем. Прежде всего это касается полноты проверки работы схемы, во многих случаях в принципе невозможной. Например, для полной проверки 16-разрядного устройства умножения потребовалось бы $2^{17}$ операций умножения и сравнения результатов с заданными значениями. При производстве даже миллиона проверок в секунду на это ушло бы 20 лет! Поэтому часто приходится ограничиваться неполной проверкой или привлекать косвенные методы. Поэтому разработка методов, которые бы при минимальном количестве проверок давали максимально возможную полноту, является весьма важной задачей.

Другая, не менее сложная проблема, - это определение неправильно работающего элемента схемы или места ошибки в монтаже, т. е. диагностика. Достижения по этой проблеме сегодня весьма скромны даже в мијровом масштабе. Имеющиеся методы либо слишком громоздки, либо требуют применения диагностических словарей большого объема. Поэтому весьма перспективной представляется методика диагностики, разрабатываемая в рамках Программы в Таллинском политехническом институте, базирующаяся на поиске места неисправностей на альтернативных графах.

В настоящее время стало правилом пользоваться детерминированными методами диагностики. При этом проверка идет с помощью заданных последовательностей кодовых комбинаций - тестов. Генерирование тестов из-за большого объема работы проводится на универсальной ЭВМ, а их передача к устройству контроля осуществляется через промежуточные носители - перфо- или магнитные ленты. Главными недостатками этого метода являются ограниченная длина тестов и сложность математического обеспечения для их генерации.

В связи с этим в последнее время интенсивное развитие получили стохастические методы контроля и диагностики, заключающиеся в том, что на вход схемы подаются случайные кодовые последовательности с заданными статистическими характеристиками. На выходе схемы определяются статистические характеристики выходных кодовых последовательностей. Так как генерация случайных последовательностей легко выполнима аппаратным путем, то отпадает надобность в применении универсальной ЭВМ и, следовательно, упрощается реализация тестов длиной в несколько миллионов комбинаций. Хотя и здесь имеется ряд 
нерешенных теоретических проблем, эти методы весьма перспективны, их разработкой также заняты наши специалисты.

При создании специализированных ЭВМ для описанных систем полностью применима как конструктивно-техническая база малых ЭВМ, так и стандартные интерфейсы с внешними устройствами.

В области эл м м н т о й базы ЭВМ ведутся работы по разработке памяти на магнитных элементах, которая наряду с полупроводниковой памятью займет определенное место и в будущем, особенно там, где требуется энергонезависимость, ибо магнитные материалы способны поддерживать свое магнитное состояние практически неограниченное время, причем без потребления энергии.

В целях получения запоминающего устройства на магнитной пленке в Институте кибернетики АН ЭССР в течение ряда лет велись исследования по электрохимическому осаждению на проволоку из бериллиевой бронзы тонкого (порядка 1 мкм) слоя ферроникелевого сплава. Была построена лабораторно-производственная линия для выявления технологических взаимосвязей кристаллической структуры подложки и покрытия, а также магнитных свойств сплава. Сконструированы специальные измерительные приборы как для применения на линии производства, так и для выявления статистических и динамических характеристик пленки с целью определения качества продукции. В настоящее время большое внимание уделяется конструктивным вопросам пленочных запоминающих устройств, в частности расчету электромагнитных характеристик матриц и созданию электронного обрамления запоминающих устройств.

Таким образом, Программа охватывает большой и сложный комплекс работ, выполнение которых требует организации вначале солидной экспериментально-производственной базы, а затем и опытного завода. В первую очередь нужен комплекс современного оборудования для изготовления печатных схем с достаточно высокой плотностью монтажа, необходимы оборудование и технология производства гибридных схем для изготовления специализированных узлов нестандартной аппаратуры (особенно для связи с различными объектами автоматизации).

Увеличения мощности требует также вычислительный центр Института кибернетики, ибо только применение ЭВМ высокой производительности позволит быстро создавать весьма большие системы программ.

Реализация рассмотренной Программы должна решить многие практические проблемы автоматизации производственных процессов и научных экспериментов как в нашей республике, так и в стране.

\section{Л И Т Е Р А Т У Р А}

1. Т ам м Б. Г. Описание языка САП-2 для программирования работы станков с ЦПУ. - В кн.: Алгоритмы и алгоритмическне языки, № 5. М., 1970, c. $67-78$.

2. Т а м м Б. Г. Новая система для металлорежущих станков. - Вестник АН СССР, 1963, № 12 , c. $42-45$.

3. Пру уден Ю. И. АПРОКС - специализированный язык с проблемной ориентацией. - В кн.: Алгоритмы и алгоритмические языки, № 5. М., 1970, с. 79-93.

4. С а р в Э. Н., Х ей н л а Л. Э. Анкетные системы обработки экономических данных. - В кн.: Вторая всесоюз. конф. по программированию. Новосибирск, 1970 , c. $77-89$.

5. T a m m, B. On the data structure and linkage problems in NC machine tool languages. - In: Numerical Control Programming Languages. IFIP/IFAC PROLAMAT Conf. Proc. North Holland. 1970, p. 342-349.

6. T a m m, B. Using NC programs and integrated CAD/CAM systems, - In: Compu- 
ter Languages for Numerical Control, IFIP/IFAC PROLAMAT Conf. Proc. North Holland, 1974, p. 133-140.

7. T a m m, B., Pru uden, J. Problem-oriented computer languages for simulating engineering processes. - In: IFIP 468 Congress Preprints, Booklet H. North Holland, 1968, p. 62-66.

8. Интегрированная система программирования (под ред. Б. Г. Тамма). Тр. ИНЭУМ, вып. 43. М., 1974.

9. Мельников И. А., М артин К. О., Прууден Э. В., Прууден Ю. И., П укк Р. А., С а а р Х. Я., Т а м м Б. Г. Метасистема для создания информационно-связанных специализированных систем программирования. - Кибернетика, 1974, № 6, с. $69-73$.

10. М а ртин К. О., Пру уден Э. В., Пру уден Ю. И., Т а м м Б. Г. Реализация общей части интегрированной системы программирования. - Программирование, 1975 , № 5 , с. 3-12.

11. T a m m, B., Pruuden, J., Pruuden, E., Märt in, K., Me Inikov, I. Integrated programming system as a tool to implement nonprocedural programming languages for engineering problems. - In: Proc. of the Finnish-Soviet Symposium «Systems Programming Methods and Tools», Tietojenkäsitelyliiton monistesarja 2/76. Espoo, 1976, p. $74-88$.

12. Т амм Б. Г., Прууден Ю И., Прууден Э. В. Автоматизация подготовки программ для металлорежущих станков с помощью ЭВМ. Таллин, 1966.

13. Т ы уг у Э. Х. Решение задач на вычислительных моделях. - ЖВМ и МФ, 1970 , т. 10, № 3 , c. $716-733$.

14. Т а в а ст Р., Я аксо о Ю. Оптимальное управление процессом производства формальдегида из метанола. - Изв. АН ЭССР, Сер. физ.-матем. и техн. наук, 1965, T. XIV. № 3, c. $444-454$.

15. T a vast, R., Mōtu s, L. An adaptive man-computer Control System for a chemical plant. - Preprints of the 4th IFAC Congress. Warszawa, 1969, p. 348-362.

16. T a vast, R. Steady-state optimizing control of a group of chemical processes. Automatica, 1972 , v. 8, N 6, p. 715-727.

17. Я аксоо Ю. И. Об адаптивном управлении безынерционным объектом. - Автоматика и вычислительная техника, 1969, № 3, с. 50-61.

18. М ы у с Л. Дифференциальные уравнения со свободными границами в задачах стохастического управления. - Изв. АН ЭССР, Физ. Матем., 1973, т. 22, № 1, c. $37-44$.

19. Т оби а с Т. Оптимальная остановка диффузионных процессов и параболические вариационные неравенства. - Дифференциальные уравнения, 1973, т. IX, № 4, c. $702-708$.

20. $\mathrm{P}$ а й к Э. В. Соотношения в задачах стохастического оптимального управления дискретными процессами. - Автоматика и телемеханика, 1971, № 1, с. 62-68.

21. $\mathrm{P}$ а й к Э. О задачах стохастического программирования с решающими функциями. - Изв. АН ЭССР, Физ. Матем., 1972, т. 21, № 3, с. $258-263$.

22. Petersen, I. Linear minimax estimation for inadequate models in $\mathrm{L}^{2}$ metric. Math. Operat. u. Statist., 1973, H. 6, N 8, S. 463-473.

23. Jaaksoo, . Design of multivariable discrete time regulators, - Preprints of the 7th IFAC World Congress. Helsinki, 1978, p. 1831-1836.

24. T a vast, R., Roose, A. Simultaneous two-level iteration procedure for chemical plant network simulation. - Trans. of the Symp. «Computers in the design and erection of chemical plants». Karlovy Vary, 1975, p. 698-706.

25. A a rna, O., Ka lil a s, J., V ibo, E. Chemical plant model generator. - Preprints of the ist IFAC/IFIP Symp. on Software for Computer Control, SOCOCO $\rightarrow 76$. Tallinn, 1976, p. 93-97.

26. H a n son, V., Mótus, L., Jaaksoo, U., T avast, R. Program system for online process control. - Preprints of the IFAC 6th World Congress. Boston, 1975, p. $413-423$.

27. Kääramees, K., Mõtus, L., Nurmela, V. A real-time executive for a chemical processs control system. - Preprints of the 1st IFAC/IFIP Symp. on Software for Computer Control, SOCOCO-76. Tallinn, 1976, p. 147-150.

28. Тинн К. А., Т ы угу Э. Х. Технологические расчеты на ЦВМ І. Л., 1968.

29. Т а м м Б. Г., Т ы у г у Э. Х. О создании проблемно-ориентированного программного обеспечения. - Кибернетика, 1975, № 4, с. $76-85$.

Ннститут кибернетики

Академии наук Эстонской ССР
Поступила в редакцию 3/V 1978 
B. TAMM, H. TANI, R. TAVAST, E. TOUGU

\section{ENSV TEADUSTE AKADEEMIA ARVUTUSTEHNIKA PROGRAMM}

Artiklis on käsitletud miniarvutite ja nende baasil ioodavate probleemorienteeritud süsteemide teaduslik-tehnilist programmi ning selle eesmärke.

Eeldused reaalajas töötavate juhtimissüsteemide '́oomiseks tekkisid Eestis 1970. aastate algul seoses 3. põlvkonna arvutite laialdasema levikuga. Projekteeriti esimesed spetsialiseeritud miniarvutid ja juhtimissüsteemid. 1976. aastal loodi Küberneetika Instituudi juurde arvutustehnika erikonstrueerimisbüroo, mis ühendab enamikku vabariigi arvutustehnikaspetsialiste ja töötab ülalnimetatud programmi järgi. Viimane näeb ette probleemorienteeritud miniarvutite projekteerimist ja teaduslik-tehnilise baasi loomist arvutite tootmiseks Eestis 11. viisaastakul.

B. TAMM, H. TANI, R. TAVAST, E. TOUGU

\section{A COMPUTER DEVELOPMENT PROGRAM OF THE ACADENIY OF SCIENCES OF THE ESSR}

The development program of minicomputers and problem-oriented systems is described and the scientific and technical aims of the program are given.

The pre-requisites for designing of real-time control systems became evident in Estonia at the beginning of the seventies in connection with the wide application of the computers of the third generation. In 1975 it was decided to unite the efforts of the computer and data processing specialists of the republic and to establish the Computer Division of the Institute of Cybernetics which began to work according to the new adopted program. The aim of the program is to design problem-oriented minicomputers and to take care of scienfitic and technical basis for the production of minicomputers in the nearest future.

The realization of the program foresees the design of certain systems, the most important of which are:

- computer-aided software design

- control systems of power generators on the basis of distributed microcomputers

- equipment for fault testing

- plated-wire memories

- automation of scientific experiment.

The program is to support computer control in technology as well as in automation of scientific experiments. 